Article

\title{
Research on Optimized Product Image Design Integrated Decision System Based on Kansei Engineering
}

\author{
Lei Xue ${ }^{1}\left(\mathbb{D}\right.$, Xiao $\mathrm{Yi}^{1}$ and Ye Zhang ${ }^{2, *}$ \\ 1 School of Mechanical, Electronic and Control Engineering, Beijing Jiao Tong University, Beijing 100044, \\ China; 13116347@bjtu.edu.cn (L.X.); xyi@bjtu.edu.cn (X.Y.) \\ 2 School of Architecture and Design, Beijing Jiao Tong University, Beijing 100044, China \\ * Correspondence: yzhang4@bjtu.edu.cn
}

Received: 18 December 2019; Accepted: 7 February 2020; Published: 11 February 2020

check for updates

\begin{abstract}
In order to facilitate the development of product image design, the research proposes the optimized product image design integrated decision system based on Kansei Engineering experiment. The system consists of two sub-models, namely product image design qualitative decision model and quantitative decision model. Firstly, using the product image design qualitative decision model, the influential design elements for the product image are identified based on Quantification Theory Type I. Secondly, the quantitative decision model is utilized to predict the product total image. Grey Relation Analysis (GRA)-Fuzzy logic sub-models of influential design elements are built up separately. After that, utility optimization model is applied to obtain the multi-objective product image. Finally, the product image design integrated decision system is completed to optimize the product image design in the process of product design. A case study of train seat design is given to demonstrate the analysis results. The train seat image design integrated decision system is constructed to determine the product image. This shows the proposed system is effective and for predicting and evaluating the product image. The results provide meaningful improvement for product image design decision.
\end{abstract}

Keywords: product image design; Kansei Engineering; integrated decision system; qualitative decision model; quantitative decision model; train seats

\section{Introduction}

The development trend of industrial design is toward more efficient, intelligent and systematic. Meanwhile, product design pays more attention to the improvement of the user experience. The development of industrial design decision system not only promotes the economic and cultural exchange between regions, but also has a profound impact on the long-term development of various related industries [1]. With the increasing demand for user experience design, user perception centered design will become an important factor affecting product design [2]. Therefore, based on meeting the product function, we should comprehensively improve the user physiological and psychological multi-dimensional emotional needs, optimize the overall product design level, promote the user experience, and raise the product design quality.

Kansei Engineering is a kind of theory based on the design science, psychology, cognition and other relevant disciplines, which can lead human perceptual analysis to the field of engineering technology [3]. Kansei Engineering not only helps designers understand users' perceptual needs, but also optimizes product design process and reduce product design cost. Kansei Engineering is a method of using engineering technology to explore the relationship between the sensibility of user and the design characteristics of product. It can transform the perceptual needs by users into the design 
elements of products. In the field of product design, Kansei Engineering is a user-oriented product research and development technology. The modern tools and technologies are used to quantify the user's perceptual information into the design parameters of product, as shown in Figure 1.

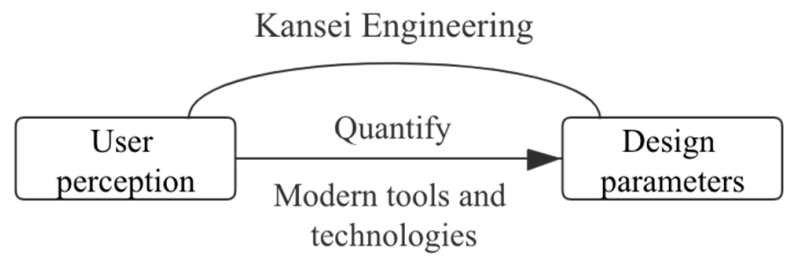

Figure 1. The Kansei Engineering of product design process.

Image is a kind of the psychological symbol that the products give to the users, and is also the psychological image or concept obtained by association and imagination. The users' cognition for the product, through the perception of five senses, causes users' resonance. Finally, a comprehensive psychological image is formed. Image has certain subjectivity and fuzziness. The product appearance design should not only meet the users' basic aesthetic needs, but also meet the users' perception needs. The basic design elements of product constitute the product appearance design. The design elements of product appearance design generally contain form, color, material, texture, pattern and so on. The formation of product image comes from the users' cognition. The design elements of product are taken as the language of communication with the user. Therefore, the formation process of product image is shown in Figure 2.

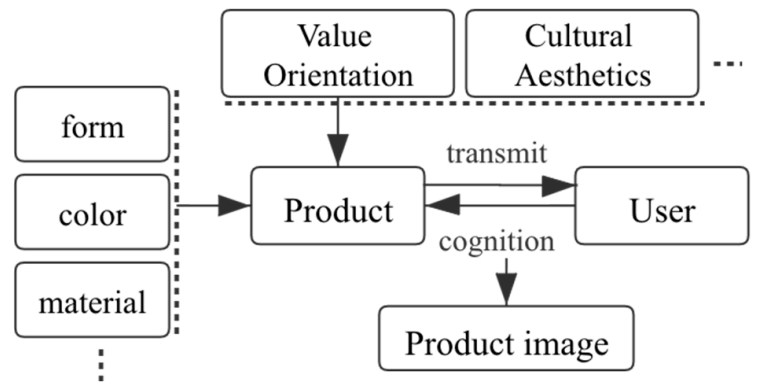

Figure 2. The formation process of product image.

Wei proposed a hybrid adaptive ant colony algorithm to realize product family multi-objective optimization design through scale-based product platform theory model [4]. Lei presented a Decision Support System (DSS) for market-driven product positioning and design, based on market data and design parameters. The proposed DSS determines market segments for new products using Principal Component Analysis, K-means classification [5]. Yang presented a decision support system based on a bi-level fuzzy computing approach that incorporates qualitative and quantitative product attributes in determining the manufacturability of a product [6]. A product design elements evaluation model was proposed, which was constructed by eye-tracking experiments, using eye movement indicators such as first gaze time, gaze order, and number of times of return, to accurately and effectively analyze product model and quantify users' emotion. The purpose of sorting the product model contribution was achieved through the weight calculation of the design elements [7]. Understanding the affective needs of customers is crucial to the success of product design. Hybrid Kansei Engineering system (HKES) is an expert system capable of generating products in accordance with the affective responses. HKES consists of two subsystems: forward Kansei Engineering system and backward Kansei Engineering system [8]. Wu proposed a preference-based evaluation-fuzzy-quantification method to determine the priority of the development of attractive factors of the product [9].

The state of the art has the following deficiencies: (1) The previous research lacks the product image design qualitative decision by the quantitative research methods; (2) It lacks the integrated 
decision system of product image design for the purpose of the total image design for assistance of the collaborative work of user, designer and expert; (3) The existing product image design model lacks the discussion of the assessment and verification with the computer-aided and 3-D printing of product design.

In our paper, we extend the research objectives to the product material design and texture design. This research proposes a new method for combining the product image design qualitative decision model and quantitative decision model. With the two models, the optimized product image design integrated decision system is constructed based on the Kansei Engineering experiment. In the process of the performance evaluation, the technology of the computer-aided and 3-D printing assist the evaluation with Kansei image of product image design integrated decision system.

Therefore, this paper explores the product image design system under the research framework of Kansei Engineering. The qualitative and quantitative research on the correlation between product design elements and user Kansei image is conducted, and the qualitative and quantitative decision models of product image design are constructed. Then, the integrated decision system of product image design is improved. The paper introduces the methodology that is divided into four steps, shown in Figure 3, including the establishment of qualitative decision model of product image design, quantitative decision model of product image design, product image design integrated decision system and its performance evaluation. Meanwhile, this paper takes the high-speed train seat design as the research object. Kansei Engineering experiment and design theory research are carried out deeply, and the more scientific, efficient and intelligent product image design evaluation system is put forward.

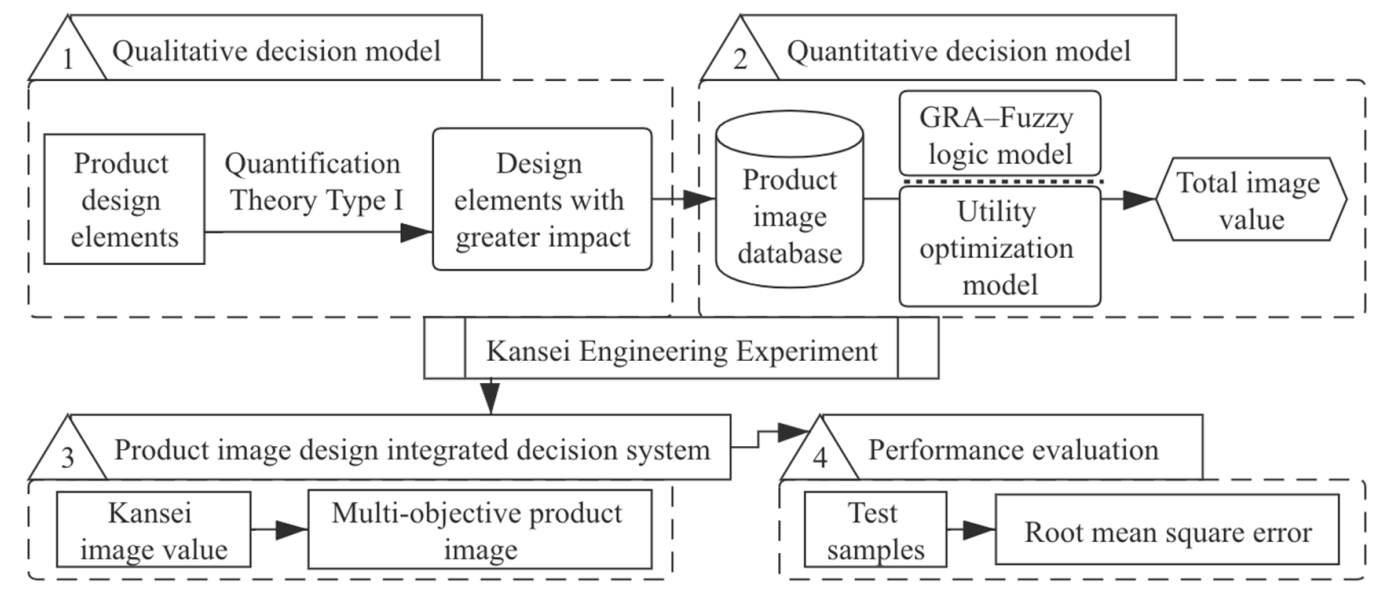

Figure 3. The four steps of the methodology process.

\section{Kansei Engineering Experiment}

The Kansei Engineering experiment process of product image design integrated decision system mainly includes design investigation stage and data statistics stage as shown in Figure 4. The design investigation stage is divided into two steps. The first step is to select the experimental subjects, experimental samples, product form samples, color samples, material samples and texture samples. Then the second step is to analyze the design elements of experimental design samples separately, such as the morphological analysis pointed to form design element.

The data statistics stage is also divided into two steps. Firstly, the Kansei word pairs are selected, including the selection of the primary Kansei words and secondary Kansei words that describe the product image. Secondly, the Kansei evaluation of the product image is carried out by the subjects for the experimental samples, and the construction of the product image database is built up through the data statistics. Based on the Kansei Engineering process, the qualitative and quantitative decision models of product image design are established to improve the product image design integrated decision system. 


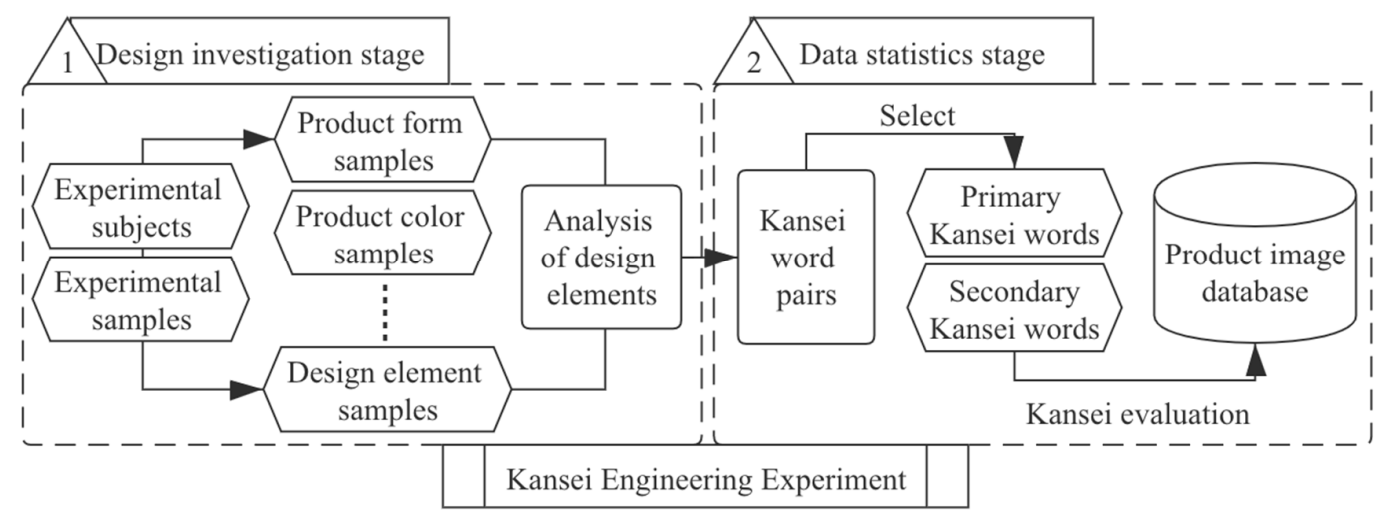

Figure 4. The Kansei Engineering experiment process of product image design.

\section{Product Image Design Integrated Decision System}

This section may be divided into product image design qualitative decision model, quantitative decision model, integrated decision system and performance evaluation. Firstly, using the Product image design qualitative decision model, the influential design elements for the product image are identified based on Quantification Theory Type I. Secondly, the quantitative decision model is utilized to predict the product total image. GRA-Fuzzy logic sub-models of influential design elements are built up separately. After that, utility optimization model is applied to obtain the multi-objective product image. Thirdly, the product image design integrated decision system is completed to optimize the product image design in the process of product design. Finally, the performance of the system is evaluated by using the root mean square error.

\subsection{Product Image Design Qualitative Decision Model}

\subsubsection{Quantification Theory Type I}

The qualitative decision model of product image design is to identify the main design elements affecting product image by quantitative analysis method. The experimental subjects in Section 2 are divided into four groups. The third group is composed of the professional product designers with rich experience in product design. Through the investigation and statistics of professional designers, the four design elements (product form, color, material and texture) are selected for further research of qualitative decision model.

Quantification Theory Type I is the method that studies the relationship between a set of qualitative variables $x$ (independent variables) and a set of quantitative variables $y$ (dependent variables) [10]. The mathematical model between variables is built up by multiple regression analysis. According to the mathematical model of Quantification Theory Type I, the procedure for establishing the Kansei-associated model is as follows:

- The mathematical formula of the Kansei-associated model is defined. The product design element is taken as the independent variable $x$ (qualitative variable) and the Kansei evaluation value is taken as the dependent variable $y$ (quantitative variable). The purpose of Quantification Theory Type I is to solve the regression coefficient of each design element [11]. Therefore, the multiple regression equation of the product design element and Kansei evaluation value can be defined as:

$$
\hat{y}_{s}^{k}=\sum_{i=1}^{D} \sum_{j=1}^{M_{i}} \beta_{i j} x_{i j s}
$$

where $k$ represents the $k$ th Kansei image, $k=1,2, \ldots, m$, and $m$ represents the number of Kansei images; $s$ represents the $s$ th experimental sample, $s=1,2, \ldots, n$, and $n$ represents the number of experimental samples; $i$ represents the $i$ th design element, $i=1,2, \ldots, D$, and $D$ represents the 
number of design elements; $j$ represents the $j$ th category of the $i$ th design element, $j=1,2, \ldots, M_{i}$, and $M_{i}$ represents the number of category for the $i$ th design element.

$\hat{y}_{s}^{k}$ is the evaluation value of the $k$ th Kansei image for the sth experimental sample. $x_{i j s}$ represents the independent variable of the $j$ th category of the $i$ th design element for the $s$ th experimental sample, so $x_{i j s}$ can be defined as follows:

$$
x_{i j s}= \begin{cases}1 & \text { the } j \text { th category of the ith design element for the sth experimental sample } \\ 0 & \text { otherwise }\end{cases}
$$

And $x_{i j s}$ meets the condition of $\sum_{j=1}^{M_{i}} x_{i j s}=1$, for $\forall i$ and $s$.

$\beta_{i j}$ represents the partial regression coefficient of the $j$ th category of the $i$ th design element.

- Using the least-squares method, error equation can be defined as below:

$$
Q=\sum_{s=1}^{n}\left(y_{s}^{k}-\hat{y}_{s}^{k}\right)^{2}
$$

where $Q$ is the sum of square error between the predicted value and the actual value. In order to improve the prediction accuracy and minimize the error, the following equation can be defined for the calculation of partial differential:

$$
\frac{\partial Q}{\partial \beta_{i j}}=0, \text { for } \forall \beta_{i j}
$$

$\beta_{i j}$ can be obtained by the simultaneous equations. Namely, the partial regression coefficient of design element can be acquired. The influence degree of each design element on the Kansei image can be obtained by analyzing the numerical value of $\beta_{i j}$.

- The complex correlation coefficient $R$ can measure the accuracy of the model. Generally, the square of the complex correlation coefficient $R$, namely, the coefficient of determination $R^{2}$ is used to represent the accuracy of the model. The coefficient of determination is also known as the contribution rate, which is used to measure the cooperation degree or interpretability of the regression equation.

- In order to measure the contribution of each product design element to the prediction evaluation of Kansei image, the partial correlation coefficient needs to be obtained. The partial correlation coefficient indicates the contribution of the $i$ th product design element to the value $y$ of Kansei evaluation. The larger the value of partial correlation coefficient is, the greater the influence of product design element on Kansei image is.

Using Quantification Theory Type I, the partial correlation coefficient of the product design element affecting the Kansei image is obtained, and then the decision coefficient of each design element category is acquired. The partial correlation coefficient represents the contribution of product design element to each Kansei image, and decision coefficient demonstrates the precision of model. In the process of product design, if the designers expect to get the higher evaluation value of specific product image, they should give priority to the product design elements with the higher partial correlation coefficient, properly ignore the design elements with the lower partial correlation coefficient, improve design efficiency, save design cost and optimize product image design process.

\subsubsection{Product Image Design Qualitative Decision Model}

As shown in Figure 5, the qualitative decision model of product image design is constructed. Firstly, the product design elements are analyzed, including product form, product color, product 
material, product texture, product pattern and other design elements. The partial correlation coefficient of product element is obtained through Quantification Theory Type I, and the priority ranking of product design elements affecting Kansei image is obtained.

The design elements for the product Kansei image with the greater impact and less impact are analyzed respectively. Designers should pay more attention to the design elements with greater impact, ignore the design elements with less impact, and build a qualitative decision model of product image design. Then, the design elements with greater influence for the profound study are selected, which provides a theoretical basis for the next product image design quantitative decision model.

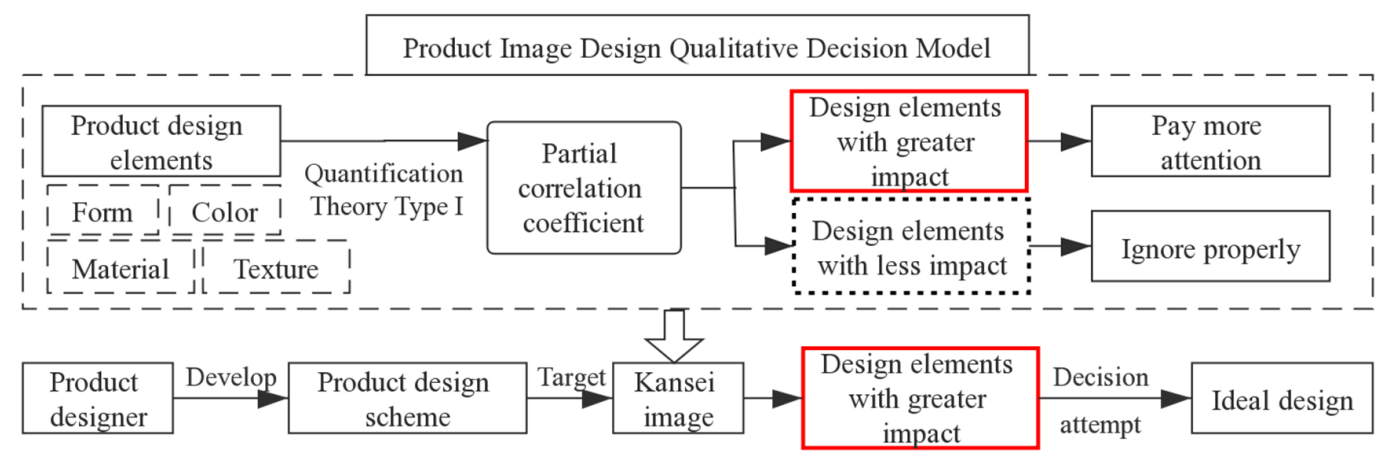

Figure 5. The qualitative decision model of product image design.

\subsection{Product Image Design Quantitative Decision Model}

\subsubsection{GRA-Fuzzy Logic Model}

Grey Relation Analysis (GRA) is a method to determine the relationship (similarity) between two sets of random sequences in grey system. One is a reference sequence $\left(x_{0} \in X\right)$ and the other is a comparison sequence $X=\left\{x_{\sigma} \mid \sigma=0,1,2, \ldots, n\right\}$, and $X=\left\{x_{\sigma} \mid \sigma=0,1,2, \ldots, n\right\}$ is a set of grey related elements [12]. At a certain moment, the grey correlation degree of two sets of series can be expressed by grey correlation coefficient $r\left(x_{0}(k), x_{i}(k)\right)$, which is defined as below:

$$
\begin{gathered}
r\left(x_{0}(k), x_{i}(k)\right)=\min _{i} \min _{k}\left|x_{0}(k)-x_{i}(k)\right|+\xi \max _{i} \max _{k}\left|x_{0}(k)-x_{i}(k)\right| / \\
\left|x_{0}(k)-x_{i}(k)\right|+\xi \max _{i} \max _{k}\left|x_{0}(k)-x_{i}(k)\right|
\end{gathered}
$$

$k=1,2, \ldots, n ; i=1,2, \ldots, m$

The grey correlation coefficients of different design sub-elements are different. If $r\left(x_{0}, x_{i}\right)>r\left(x_{0}, x_{j}\right)$, then design sub-element $x_{i}$ is more relevant to product image than design sub-element $x_{j}$. Based on the Kansei image database of each design sub-element affecting the product image, the grey correlation coefficient is used to express the relationship between the product image and design sub-elements. By comparing the numerical value of grey correlation coefficient, the sorting of the influence degree of different design sub-elements on product image is obtained. The most influential design sub-elements are selected as input linguistic variables, and the corresponding product image as output linguistic variable of GRA-Fuzzy logic model.

Based on the GRA-Fuzzy theory, combining with the Kansei Engineering experiment, the product design sub-elements with the greater influence are selected first, and the product image of the experimental samples is evaluated by seven-point Likert scale [13]. Then, the influential design sub-elements are taken as the input fuzzy set, and the evaluation values of product image are taken as the output fuzzy set. The triangular fuzzy functions of input and output linguistic variables are obtained. Through the application of fuzzy logic model in MATLAB, the fuzzy rules are constructed, and the center of maximum (CoM) method is used for defuzzification [14]. Finally, the GRA-Fuzzy logic model of product image design is established to determine the specific Kansei image value of product design. 
Combined with the product image database in Section 2, the fuzzy rules of GRA-Fuzzy logic model are constructed. In order to reflect the relationship between the multiple design sub-elements and product images involved in the product design process, the "if ... then ... " rules with multiple fuzzy conditions are used as follows:

$$
\begin{aligned}
& \text { If } X_{1} \text { is } A_{1} \text { and } X_{2} \text { is } A_{2} \cdots \text { and } X_{n} \text { is } A_{n} \text {; } \\
& \text { Then } Y_{1} \text { is } B_{1} \text { and } Y_{2} \text { is } B_{2} \cdots \text { and } Y_{n} \text { is } B_{n}
\end{aligned}
$$

where $A_{1}, A_{2}, \ldots, A_{n}$ and $B_{1}, B_{2}, \ldots, B_{n}$ are fuzzy linguistic terms, which are, respectively, represented by input linguistic variables $X_{1}, X_{2}, \ldots, X_{n}$ and output linguistic variables $Y_{1}, Y_{2}, \ldots, Y_{n}$. The input linguistic variables $X_{1}, X_{2}, \ldots, X_{n}$ represent sub-elements of product design, and the output linguistic variables $Y_{1}, Y_{2}, \ldots, Y_{n}$ represent product image. Each fuzzy rule of GRA-Fuzzy logic model of product image design is to associate the given combination of product design sub-elements with the corresponding numerical state of product image.

Defuzzification is the process of transforming the membership of output linguistic variables into numerical value. The most commonly used defuzzification technology, the center of maximum (CoM), is determined as follows:

$$
y \operatorname{CoM}=\frac{\sum_{i}\left[\mu\left(y_{i} \times y_{i}\right)\right]}{\sum_{i} \mu\left(y_{i}\right)}
$$

where $i$ represents the linguistic item of output linguistic variable, $y_{i}$ is the maximum value of each linguistic item $i$, and $\mu\left(y_{i}\right)$ is the aggregate output membership function.

Finally, the GRA-Fuzzy logic model of product image design is constructed to assist designers to evaluate the product image design. When the product designers input the combination of product sub-elements, they can output the value of product image and obtain the numerical states of multiple product images.

\subsubsection{Utility Optimization Model}

In the product design process, it is difficult to judge design scheme by using a single index. For the solution method of multi-objective programming, the utility optimization model is generally used through transforming the multi-objective model into the single-objective model. The model solution basic idea is that each objective function of the programming problems can be calculated in a certain way [15].

$$
\begin{aligned}
& \max Z=\psi(X) \\
& \text { s.t. } \Phi(X) \leq G
\end{aligned}
$$

$\psi$ is the sum function of utility functions related to each objective function.

When using utility function as planning objective, it is necessary to determine a set of $w_{i}$ that reflects the weight of each objective function in the overall objective of the original problem, namely:

$$
\begin{gathered}
\max \psi=\sum_{i=1}^{k} w_{i} \psi_{i} \\
\Phi\left(x_{1}, x_{2}, \ldots, x_{n}\right) \leq g_{i}(i=1,2, \ldots, m)
\end{gathered}
$$

where $w_{i}$ should satisfy $\sum_{i}^{k} w_{i}=1$

Parametric vector form is shown as below:

$$
\max \psi=w^{T} \psi
$$




$$
\text { s.t. } \Phi(X) \leq G
$$

\subsubsection{Product Image Design Quantitative Decision Model}

The quantitative decision model of product image design is constructed as shown in Figure 6. Based on the Kansei Engineering experiment, the qualitative decision model of product image design in Section 3.1.2 is used to get the influential design elements for product image, and the GRA-Fuzzy logic sub-models of the influential design elements are constructed respectively.

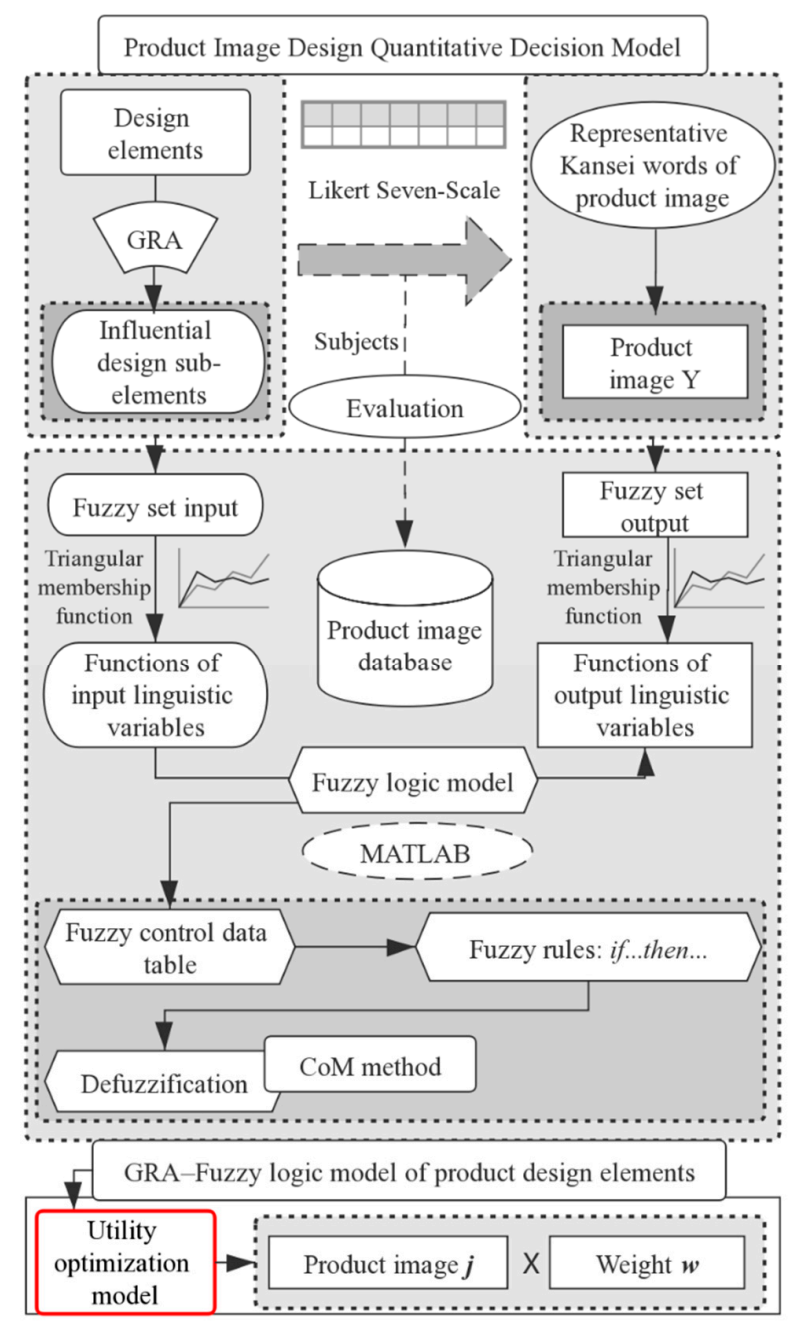

Figure 6. The quantitative decision model of product image design.

Firstly, using the Kansei Engineering experiment process, the design elements with great influence are analyzed, and the sub-elements of the influential design elements are obtained. Meanwhile, the representative Kansei words of the product image expected by users are determined. The subjects evaluate the product image of the experimental samples by Likert scale to set up the product image database. Then, through the grey relational analysis (GRA), the grey correlation coefficients of the design sub-elements that affecting the product image are obtained, and the numerical values of the grey correlation coefficients are sorted and compared in order to obtain the design sub-elements that have a great influence on the product image.

The design sub-elements with great influence obtained above are taken as input linguistic variables, and the product image expected by users is taken as an output linguistic variable. The fuzzy functions of input linguistic variables and output linguistic variables are constructed by the function of triangular membership, and the GRA-Fuzzy logic model is constructed by MATLAB. The fuzzy control data 
table and the corresponding fuzzy rules between design sub-elements and product image are obtained. Then, the fuzzy numbers are defuzzified by using the CoM method. Finally, the GRA-Fuzzy logic sub-models of the influential design elements are established.

Users usually expect to obtain multiple product images in the practical design and production process. The utility optimization model is used to transform the multi-objective product image into the single-objective product image, optimize the GRA-Fuzzy logic model of product design elements. The weights of product image are obtained according to the demand of design objectives, which reflects the weight of each function of product image in total product image objective, and the product total image value is obtained by linear weighting method. The quantitative decision model of product image design is optimized, namely:

$$
g(x)=\sum_{j=1}^{q} w_{j} g_{j}(x)
$$

where $g_{j}(x)$ is the GRA-Fuzzy logic model of product image $j . w_{j}$ is the weight of the $j$ th product image function $g_{j}(x)$ obtained according to the requirements of objective programming in the product total image objective.

The GRA-Fuzzy logic sub-models of product design elements can help designers evaluate the design elements of product image. Based on the construction of GRA-Fuzzy logic model and utility optimization model, the product image design model is further optimized, and the quantitative decision model of product image design is constructed.

\subsection{Product Image Design Integrated Decision System}

As shown in Figure 7, product image design integrated decision system is composed of qualitative decision model and quantitative decision model. For the varied kinds of industrial product design, firstly, the qualitative decision model of product image design is constructed to obtain the product design elements that have a greater influence on the user's Kansei image. After that, the quantitative decision model of product image design is implemented to acquire the relationship between the influential design sub-elements and the numerical value of Kansei image. The collaborative work of the above two models constitutes the integrated decision system of product image design. Therefore, it is more efficient and accurate that the combination of product design elements matches with the output of user's Kansei image.

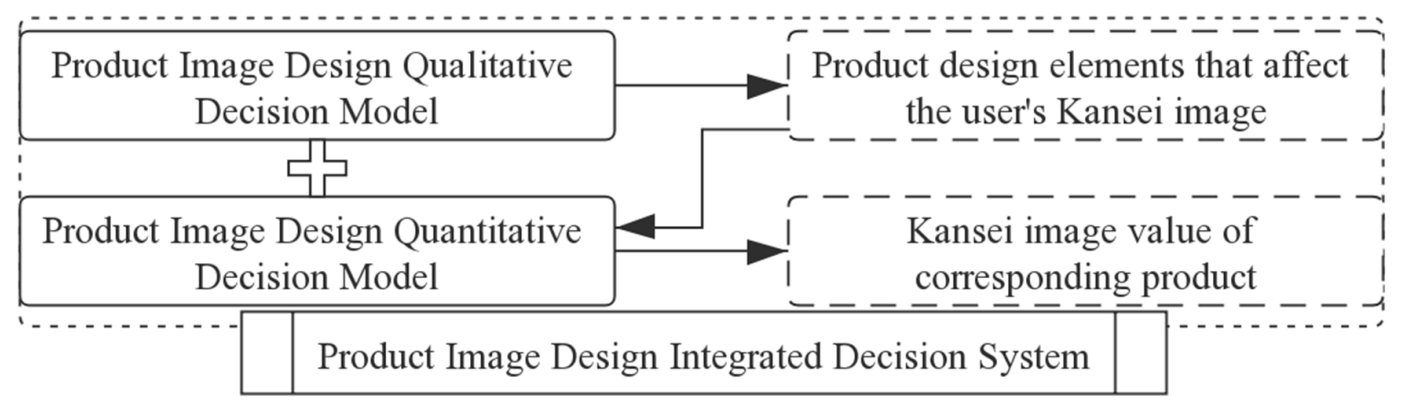

Figure 7. The product image design integrated decision system.

\subsection{Performance Evaluation}

In order to evaluate the performance of the product image design integrated decision system, the root mean square error (RMSE) is used as follows [16]:

$$
R M S E=\sqrt{\frac{\sum_{i=1}^{n}\left(x_{i}-x_{0}\right)^{2}}{n}}
$$


where $x_{i}$ is the $i$ th output value of product image design integrated decision system and $x_{0}$ is the expected value evaluated by the experimental subjects. We use test samples to evaluate the prediction performance of product image design integrated decision system, and the subjects of the second group evaluate the product total image of test samples to get the numerical value. Then, the design sub-elements of each sample are input into the product image design integrated decision system, and the output value of the product total image is obtained, which compared with the evaluation value of the subjects. The RMSE of product image design integrated decision system is obtained. Then, the performance evaluation of product image design integrated decision system is completed.

The case study shows that the optimized product image design integrated decision system has a good performance for predicting and evaluating the product image. The proposed system has a better prediction ability of Kansei image for the new product with given design elements.

\section{Case Study of Train Seat Image Design}

Based on the research of the optimization process of the product image design integrated decision system in Section 3, this section aims at optimization of the high-speed train seat image design decision system. The qualitative and quantitative decision models of the high-speed train seat image design are constructed to further promote the process of the train seat image design integrated decision system.

\subsection{Kansei Engineering Experiment}

\subsubsection{Design Investigation Stage}

Firstly, the experimental subjects and samples are selected. Afterwards, the final experimental samples are determined by the selection of train seat form, color, material and texture. Finally, the representative Kansei image words for describing the train seat image are chosen.

- Experimental subject selection

The experimental study involves 60 subjects, divided into four groups. The first group includes general users (10 males and 10 females). In the second group, there are expert users (10 males and 10 females). The third group is composed of 10 professional product designers (6 males and 4 females), and the designers of this group have at least 6 years of product design experience on average. The fourth group includes 10 experts ( 5 males and 5 females) in the field of high-speed train.

- Experimental sample selection

The subjects of the first group classify the selected product pictures based on their similarity degree of appearance design of train seat, using the Kawakita Jiro (KJ) method. Therefore, the 20 subjects of the first group classify 198 seat pictures of different manufacturers and models that entered the market from 2009 to 2019. Based on the classification results of the subjects, 30 representative experimental samples of train seats are selected by multi-dimensional scale analysis and cluster analysis.

- Design element analysis

The train seat form samples are extracted from 30 representative seat samples. According to the professional knowledge and design experience, 10 designers of the third group obtain the four design elements (including the seat form, color, material and texture) for future analysis. After that, they are required to extract seat form samples through similarity classification, and then 10 subjects are organized into focus groups to summarize and integrate their research results. After that, the 6 form samples are obtained, which are divided into three groups on average, the first group is the form experimental samples. The form experimental sample of train seat No. 2 of the first group is shown in Figure 8. 

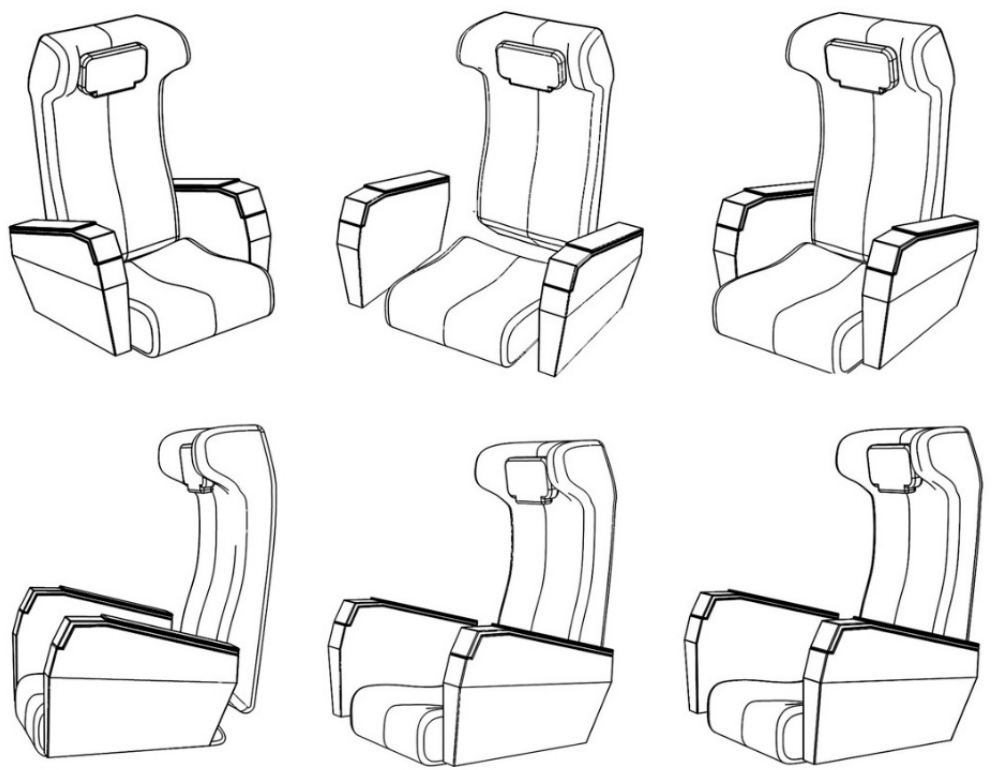

Figure 8. The form experimental sample of high-speed train No. 2 of the first group.

This study regards a small amount of color as a huge work of color speculation and establishes the relationship between product form, color, material, texture and product image [17]. The 10 subjects are organized into a focus group to conduct the inductive integration and analysis of color selection. Finally, the 6 color samples are selected as the color experimental samples of train seat. The form samples are filled with the 6 color experimental samples respectively. The form sample No. 2 is filled with the 6 color experimental samples in Figure 9 as below.
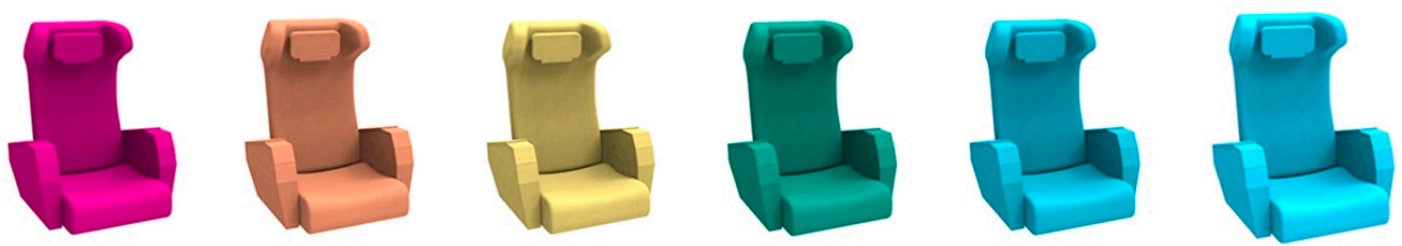

Figure 9. The 6 color experimental samples of the train seat form sample No. 2.

The subjects of third group analyzed the material design and texture design of the train seat, and the representative material samples and texture samples of train seats are collected and selected by the third subjects, and the material samples and texture samples are applied to the color samples. Through the research and investigation, the representative material samples are divided into cotton fabric, wool fabric, chemical fiber and leather, as shown in Table 1.

Table 1. The 4 material experimental samples of the train seat.

\begin{tabular}{lllll}
\hline Cotton Fabric & Wool Fabric & Chemical Fiber & Leather \\
\hline & & & \\
\hline
\end{tabular}

The representative texture samples are divided into coarse texture and fine texture. The above 4 material samples and 2 texture samples are applied to 12 train seat samples in the first group, a total of 96 train seat experimental samples, as the final experimental samples of this study. 
- Kansei image word selection

Based on the preceding steps, three groups of representative Kansei words are determined to describe the Kansei image of train seat: traditional-modern, simple-complex, unique-ordinary. We use "traditional-modern" (T-M) as the primary Kansei word of the train seat, which is the target image for constructing the qualitative decision model and quantitative decision model of the seat image design. The above experimental process becomes the application case for developing the method of Kansei Engineering.

\subsubsection{Data Statistics Stage}

The expert users evaluate the product image matched with the seat samples. As shown in Figure 10, semantic difference method is used to make the seven-point Likert scale, and 20 subjects are required to carry out the numerical value evaluation of T-M product image for the experimental samples ( 1 and 7 represent the extremely traditional and extremely modern image respectively). For example, seat No. 2 has "modern" image, namely that the maximum value of T-M image is 7 , the minimum value is 3 , and the average value is 5.33. The data statistics stage provides the experimental database for the qualitative decision model and quantitative decision model of seat image design.

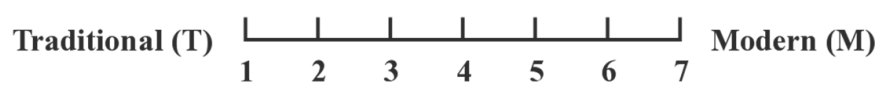

Figure 10. The seven-point Likert scale of T-M product image.

\subsection{Train Seat Image Design Qualitative Decision Model}

Through the analysis of Quantification Theory Type I, the importance and influence degree of train seat design elements are obtained. The independent variables (such as seat form, color, material and texture) of factors quantification are the nominal scales, so the numerical values of 1,2,3 and 4 are used to represent the categories of design elements, for example, "cotton fabric", "wool fabric", "chemical fiber" and "leather" of material samples are, respectively, represented by 1, 2, 3 and 4 . The result is shown in Table 2.

Table 2. Analysis of result from Quantification Theory Type I.

\begin{tabular}{ccc}
\hline Design Element & Standard Coefficient & Partial Correlation Coefficient \\
\hline Form element $\left(X_{1}\right)$ & 0.290 & 0.441 \\
Color element $\left(X_{2}\right)$ & 0.769 & 0.791 \\
Material element $\left(X_{3}\right)$ & -0.205 & -0.290 \\
Texture element $\left(X_{4}\right)$ & 0.002 & 0.003 \\
Constant & 2.464 & \\
Coefficient of determination $R^{2}$ & 0.655 & \\
\hline
\end{tabular}

The partial correlation coefficients of design elements in Table 2 show that the correlation between four independent variables (seat form, color, material and texture) and Kansei image words (T-M). For example, for the T-M image, the partial correlation coefficients of design elements are $0.441,0.791$, 0.290 and 0.003 , respectively, $X_{2}$ of which is the highest, indicating that the "color" factor is the design factor that has the greatest influence on T-M image of the independent variables. The second factor is seat form $\left(\mathrm{X}_{1}\right)$, which shows that when the design expectation of the train seat is to obtain the T-M image, the designer should pay more attention to color design element and form design element. On the contrary, designers can properly ignore the design elements with less relevance, such as seat material $\left(X_{3}\right)$ and texture $\left(X_{4}\right)$, because these two design elements have a relatively small impact on T-M image.

Based on the above experimental process of Kansei Engineering, the qualitative decision model of train seat image design is finally obtained by using Quantification Theory Type I method. The qualitative 
decision model of train seat image design is shown in Figure 11. The partial correlation coefficients of seat design elements are obtained through Quantification Theory Type I. The sequence of design elements affecting the Kansei T-M image of train seat is color, form, material and texture. Therefore, the study selects the design elements that have a great influence on the Kansei image: seat form and seat color, which provides a theoretical basis for constructing the quantitative decision model of train seat image design in the next step.

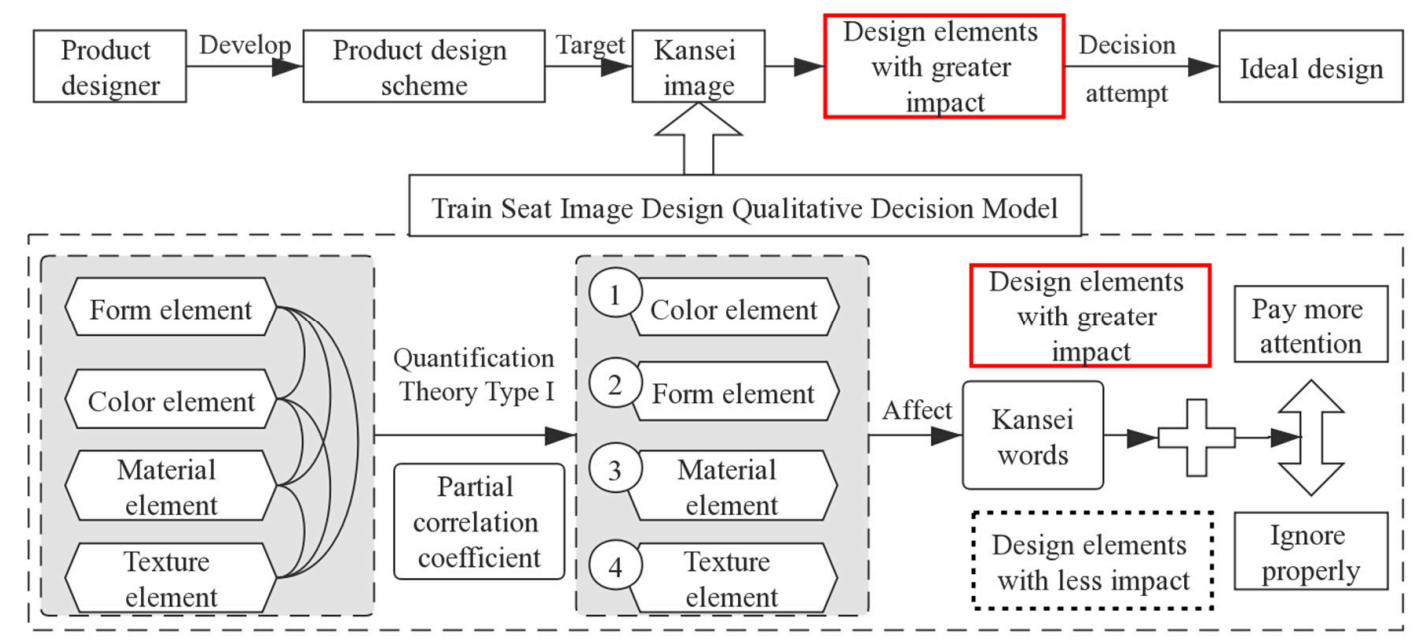

Figure 11. The qualitative decision model of train seat image design.

\subsection{Train Seat Image Design Quantitative Decision Model}

Based on the conclusion of train seat image design qualitative decision model, the design elements that have great influence on seat image are seat form and color design elements. Using the experimental process of Kansei Engineering, the GRA-Fuzzy logic sub-models of train seat form design and color design are respectively constructed. Based on the GRA-Fuzzy theory, this paper analyzes the form sub-elements and the color sub-elements. Aimed at the representative Kansei words of the seat image, the subjects evaluate the Kansei image of seat samples through the seven-point Likert scale to build up the train seat image database.

As the result of design element analysis, the 9 form sub-elements and their corresponding element types are extracted from the 30 representative train seat samples through morphological analysis. In this section, we select one representative train seat randomly from the 30 representative train seat samples and fill the selected train seat with 72 representative colors as the experimental train seat color samples, for a total of 72 color samples. In Table 3, Column 1 and 7 display the numbers of color samples, and the remaining columns display the corresponding color sub-element ("Hue $\left(h^{\circ}\right)$ " element $\left(x_{c 1}\right)$, "Chroma $\left(C^{*}\right)^{\prime \prime}$ element $\left(x_{c 2}\right)$, “Lightness $\left(L^{*}\right)$ " element $\left(x_{c 3}\right)$, "parameter $a^{* \prime}$ element $\left(x_{c 4}\right)$ and "parameter $b^{* \prime}$ element $\left.\left(x_{c 5}\right)\right)$ values of these color samples.

Table 3. Color element values of the representative train seat color sample C22-C33.

\begin{tabular}{llllllllllll}
\hline No. & $\boldsymbol{h}^{\circ}$ & $\boldsymbol{C}^{*}$ & $\boldsymbol{L}^{\boldsymbol{*}}$ & $\boldsymbol{a}^{\boldsymbol{*}}$ & $\boldsymbol{b}^{\boldsymbol{*}}$ & No. & $\boldsymbol{h}^{\circ}$ & $\boldsymbol{C}^{*}$ & $\boldsymbol{L}^{*}$ & $\boldsymbol{a}^{*}$ & $\boldsymbol{b}^{*}$ \\
\hline C22 & 90 & 60 & 50 & 0 & 60 & C28 & 135 & 60 & 30 & -42 & 42 \\
C23 & 90 & 40 & 50 & 0 & 40 & C29 & 135 & 40 & 30 & -28 & 28 \\
C24 & 90 & 20 & 50 & 0 & 20 & C30 & 135 & 20 & 30 & -14 & 14 \\
C25 & 90 & 60 & 70 & 0 & 60 & C31 & 135 & 60 & 50 & -42 & 42 \\
C26 & 90 & 40 & 70 & 0 & 40 & C32 & 135 & 40 & 50 & -28 & 28 \\
C27 & 90 & 20 & 70 & 0 & 20 & C33 & 135 & 20 & 50 & -14 & 14 \\
\hline
\end{tabular}

The GRA method is used to obtain the grey correlation degree of form design sub-element as shown in Table 4 from Section 3.2.1, and the sub-elements of form design that affect the Kansei image 
of train seat are, respectively, "Seat back waistline" $\left(x_{5}\right)$, "Seat back body shape" $\left(x_{4}\right)$, "Headrest shape" $\left(x_{2}\right)$, "Length ratio between seat-back to seat" $\left(x_{8}\right)$, "Relationship between seat-back and headrest" $\left(x_{3}\right)$ and "Seat-back slope angle" $\left(x_{9}\right)$. The above form design sub-elements that have greater influence on Kansei image are taken as the input linguistic variables and the Kansei image of train seat as output linguistic variables. The GRA-Fuzzy logic sub-model between the form design sub-elements and Kansei image is constructed by the triangular membership function.

Table 4. The numerical values of $r\left(x_{0}, x_{i}\right)$.

\begin{tabular}{ccc}
\hline \multicolumn{3}{c}{ Grey Correlation Degree } \\
\hline$r\left(x_{0}, x_{1}\right)=0.722$ & $r\left(x_{0}, x_{2}\right)=0.812$ & $r\left(x_{0}, x_{3}\right)=0.794$ \\
$r\left(x_{0}, x_{4}\right)=0.833$ & $r\left(x_{0}, x_{5}\right)=0.844$ & $r\left(x_{0}, x_{6}\right)=0.515$ \\
$r\left(x_{0}, x_{7}\right)=0.772$ & $r\left(x_{0}, x_{8}\right)=0.798$ & $r\left(x_{0}, x_{9}\right)=0.787$ \\
\hline
\end{tabular}

Afterwards, the corresponding 54 fuzzy rules are built up, and the GRA-Fuzzy logic sub-model of train seat form design is constructed. The fuzzy rules No. 22-No. 30 have been added as followed in Table 5.

Table 5. The fuzzy rules No.22-No.30 of GRA-Fuzzy logic sub-model of form design sub-elements.

\begin{tabular}{ccccccccc}
\hline & \multicolumn{9}{c}{ Rule No. } & \multicolumn{1}{c}{ If } & \multicolumn{3}{c}{ Then } \\
\cline { 2 - 10 } & $x_{2}$ & $x_{3}$ & $x_{4}$ & $x_{5}$ & $x_{8}$ & $x_{9}$ & Y(T-M) & Dos \\
\hline 22 & I & J & PL & AL & MR & SA & M & 0.75 \\
23 & I & J & IC & AL & SR & LA & M & 0.58 \\
24 & I & J & IC & AL & SR & LA & VM & 0.42 \\
25 & E & I & RC & IL & MR & LA & VM & 0.83 \\
26 & E & I & RC & IL & MR & LA & M & 0.17 \\
27 & E & S & PL & IL & SR & SA & M & 0.83 \\
28 & E & S & PL & IL & SR & SA & N & 0.17 \\
29 & S & I & RC & PL & SR & SA & N & 0.75 \\
30 & S & I & RC & PL & SR & SA & M & 0.25 \\
\hline
\end{tabular}

According to Section 3.2.1, GRA method is used to obtain the grey correlation degree of color design sub-element. It shows that the color design sub-elements that affect the Kansei image of train seat are "Lightness $\left(L^{*}\right)^{\prime \prime}\left(x_{c 3}\right)$, "Chroma $\left(C^{*}\right)^{\prime \prime}\left(x_{c 2}\right)$, "parameter $a^{* \prime}\left(x_{c 4}\right)$ and "parameter $b^{* \prime \prime}$ $\left(x_{c 5}\right)$. Taking the above color design sub-elements that have great influence on seat Kansei image as input linguistic variables and seat Kansei image as output linguistic variables, the GRA-Fuzzy logic sub-model of color design sub-elements matched with Kansei image is constructed by triangular membership function. Next, the 144 corresponding fuzzy rules are established, and the GRA-Fuzzy logic sub-model of train seat color design is constructed.

Based on the cooperative work of GRA-Fuzzy logic sub-models of the aforementioned train seat form design and color design, in terms of the application of utility optimization model, the utility optimization model is constructed for the Kansei image output value of train seat for perceptual cognition of users. Three groups of representative Kansei words are selected, including traditional-modern (T-M), simple-complex (S-C) and unique-ordinary (U-O).

In accordance with the method of multi-image objective programming, three corresponding GRA-Fuzzy logic sub-models of T-M, S-C and U-O images are established, respectively. When using utility function as the planning objective, three corresponding weights $w_{1}, w_{2}$ and $w_{3}$ are used to reflect the weight of each GRA-Fuzzy logic sub-model of each Kansei image in the overall objective, and the utility optimization model of the product total image is obtained. When the primary image of seat design expected by users is T-M image, followed by S-C and U-O image, according to the planning of users' perceptual needs, the weight of GRA-Fuzzy logic sub-model for T-M image is 0.4, and the 
weight of GRA-Fuzzy logic sub-model reflecting S-C image and U-O image is 0.3, respectively. Finally, the seat design total image of multi-image planning objective of train seat is obtained.

Based on the experiment process of Kansei Engineering, combined with the utility optimization model, the seat image design quantitative decision model of high-speed train is finally constructed, and the specific process is shown in Figure 12.

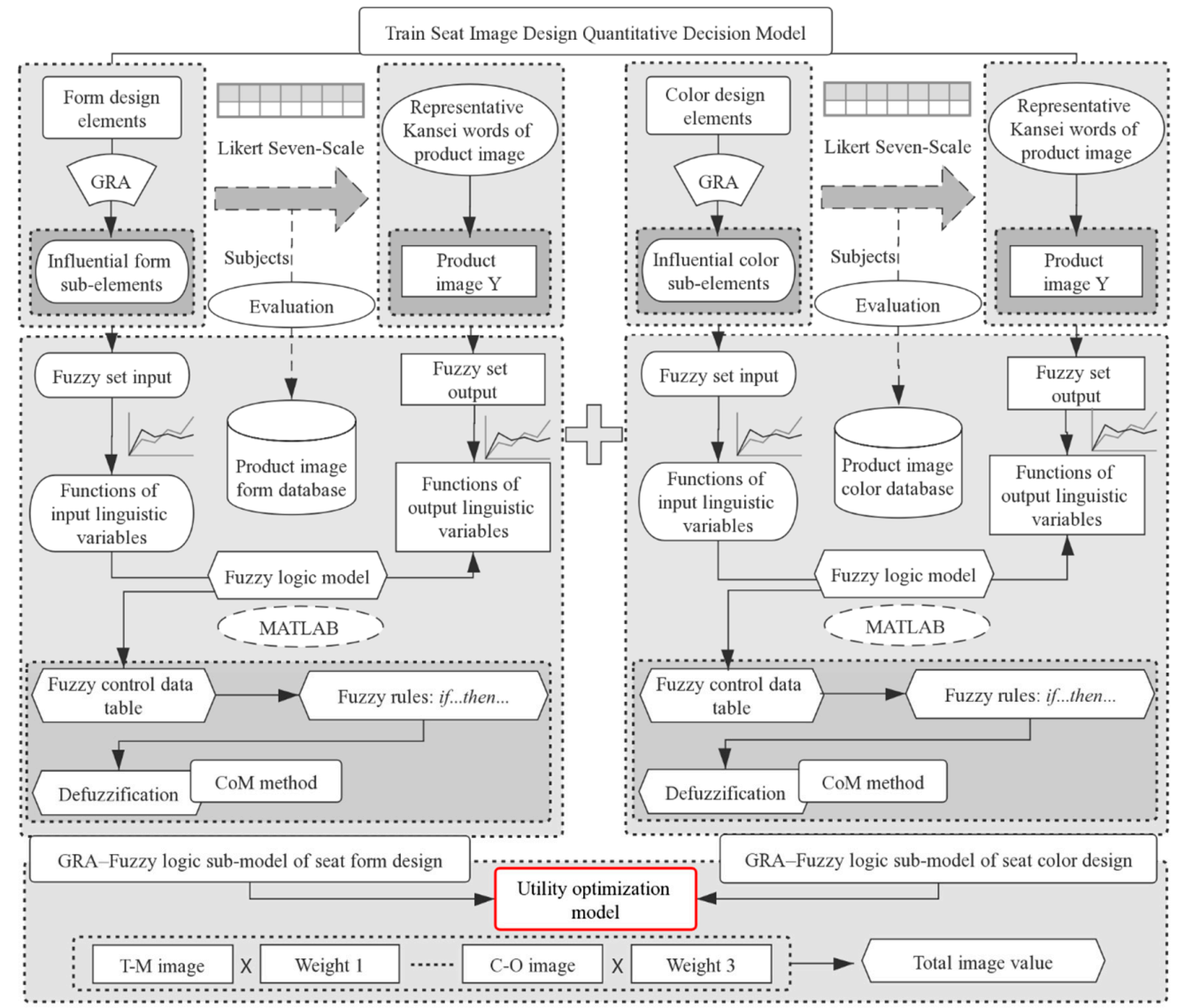

Figure 12. The quantitative decision model of train seat image design.

\subsection{Train Seat Image Design Integrated Decision System}

As shown in Figure 13, the train seat image design integrated decision system consists of qualitative decision model and quantitative decision model of seat image design. Aiming at the seat image design of high-speed train, the qualitative decision model of the seat image design is constructed firstly, and then the seat form design elements and color design elements that affect the Kansei image are obtained. After that, the quantitative decision model of the seat image design is constructed to obtain the predicted numerical value of the total image of the corresponding seat. The collaborative work of the above two models constitutes the integrated decision system of train seat image design, which makes the image design process of train seat more intelligent, accurate and efficient.

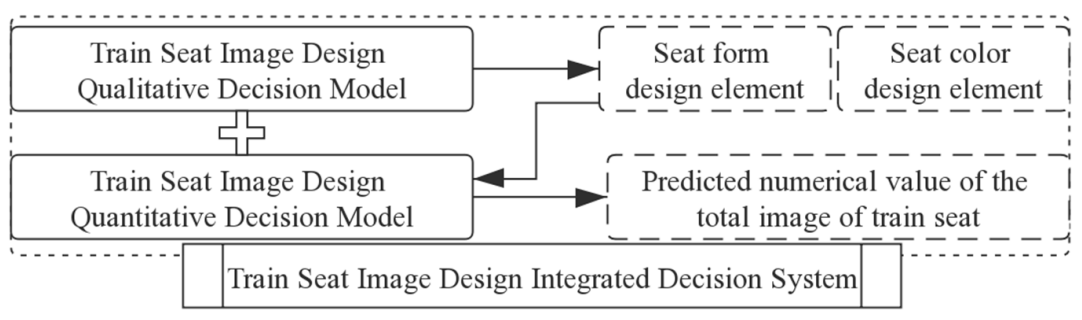

Figure 13. The train seat image design integrated decision system. 


\subsection{Performance Evaluation}

In order to verify the prediction ability of train seat image design integrated decision system, five test samples shown in Table 6 are used, including form design sub-elements and color design sub-elements that have greater influence on product image. The performance of train seat image design integrated decision system is evaluated by using the root mean square error.

Table 6. Input and output values of the seat test samples.

\begin{tabular}{cccccccccccc}
\hline Sample & $x_{2}$ & $x_{3}$ & $x_{\mathbf{4}}$ & $x_{5}$ & $x_{\mathbf{8}}$ & $x_{\mathbf{9}}$ & $x_{\mathrm{c} 2}$ & $x_{\mathrm{c} 3}$ & $x_{\mathrm{c} 4}$ & $x_{\mathrm{c} 5}$ & Y(T-M) \\
\hline 1 & SE & S & RC & CL & MR & SA & 20 & 30 & -14 & 14 & 3.90 \\
2 & I & J & IC & CL & MR & LA & 60 & 70 & 0 & -60 & 6.35 \\
3 & E & S & IC & IL & MR & LA & 60 & 30 & -42 & 42 & 4.31 \\
4 & I & S & IC & IL & MR & LA & 20 & 70 & 0 & 20 & 4.00 \\
5 & SE & I & PL & FL & SR & SA & 60 & 50 & 0 & -60 & 4.73 \\
\hline
\end{tabular}

We select T-M image as the primary image of seat design, then $\mathrm{S}-\mathrm{C}$ and $\mathrm{U}-\mathrm{O}$ image as the secondary image, and apply the seat image design integrated decision system to predict the product total image. The second row of Table 7 shows the average value of the product total image evaluated by the 20 subjects of second group on five test samples, and meanwhile, the third row indicates the prediction value of the integrated decision system of seat image design. The results show that the seat image design integrated decision system has lower RMSE value. Therefore, the integrated decision system of seat image design provides an effective mechanism for train seat design to match with the total image of product design expected by users.

Table 7. RMSE result of the integrated decision system of train seat total image design.

\begin{tabular}{ccccccc}
\hline Total Image & Sample 1 & Sample 2 & Sample 3 & Sample 4 & Sample 5 & RMSE \\
\hline Evaluation by subjects & 4.19 & 5.69 & 4.22 & 4.07 & 4.67 & \\
Integrated decision system & 4.40 & 5.48 & 4.26 & 4.34 & 4.54 & 0.1895 \\
\hline
\end{tabular}

The good performance of the seat image design integrated decision system shows that the system can help the designer to evaluate the combination of train seat design elements, aiming at satisfying the expected Kansei image. Therefore, this paper provides an effective design evaluation system for the improvement of seat design reflecting the ideal product image through the product image design integrated decision system. For example, the product designer can input the values of multiple design sub-elements by using the product image design integrated decision system, and then the predicted value of multi-objective product image is obtained. If the predicted value does not meet the standard of designer, the corresponding new product image predicted value can be obtained by modifying the combination of design sub-elements until the designer obtains the satisfactory product image value.

\subsection{Computer-Aided Seat Design and Image Evaluation}

Combined with the computer-aided seat form design and color design, the seat form and color design elements are input into the train seat image design integrated decision system, and then the product image value is obtained. Through the further application of the computer-aided product form modeling and color rendering, train seat image design integrated decision system is verified for the predictable performance of product image.

Based on the computer-aided seat test sample form design and color design, four color design samples are applied to three form design samples, and a total of 12 product design samples are obtained. Figure 14 shows the seat rendering of the form sample 3 filled with the color sample 1. 


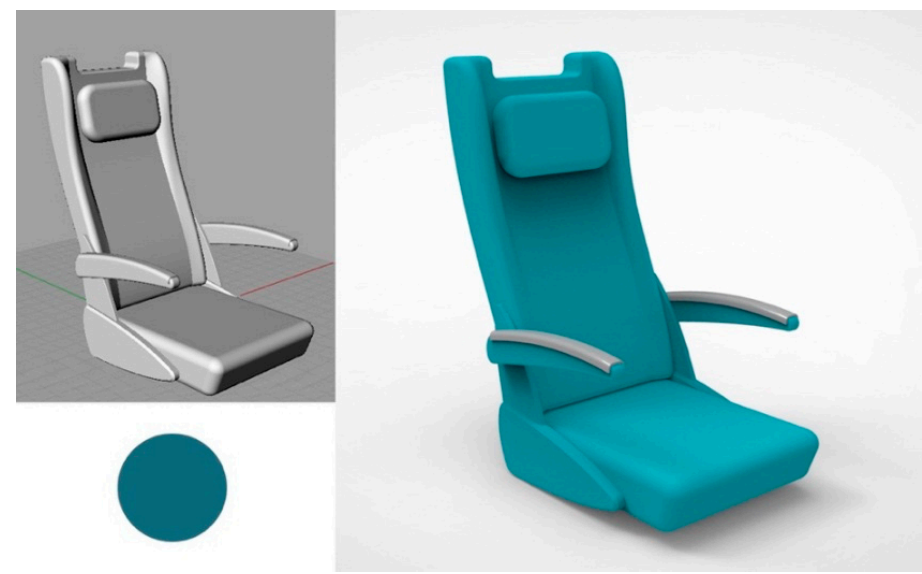

Figure 14. The train seat rendering of the form sample 3 filled with the color sample 1.

At first, the experimental subjects are required to evaluate the T-M image of the above 3-D seat models. After that, the T-M image outputs of the seat models are carried out by using the train seat image design integrated decision system. The T-M image results of models 5 to 10 are shown in the third row of Table 8. Finally, the RMSE value of the train seat image design integrated decision system is calculated with the result of less than 0.5 , which proves that integrated decision system of train seat image design performs better in predicting the $\mathrm{T}-\mathrm{M}$ image.

Table 8. RMSE result of the integrated decision system of train seat T-M image design.

\begin{tabular}{cccccccc}
\hline T-M Image & Model 5 & Model 6 & Model 7 & Model 8 & Model 9 & Model 10 & RMSE \\
\hline Evaluation by subjects & 3.86 & 5.12 & 4.84 & 5.92 & 3.22 & 5.12 & \\
Integrated decision system & 4.03 & 5.45 & 5.35 & 5.69 & 3.53 & 4.95 & 0.3098 \\
\hline
\end{tabular}

In conclusion, the integrated decision system of seat image design can effectively predict the image value of 3-D seat model of computer-aided design and rendering. Therefore, utilizing computer software to build 3-D model of industrial product, combined with computer-aided 3-D printing rapid prototyping, we can assist the use of product image design integrated decision system, which makes the image evaluation of product design more intuitive and efficient. Figure 15 illustrates the 3-D printed drawing of the form sample 3. In this study, 3-D model printing is carried out according to the ratio of 5:1 reduction of seat 3-D model to actual size in order to assist the evaluation process of integrated decision system of seat image design.

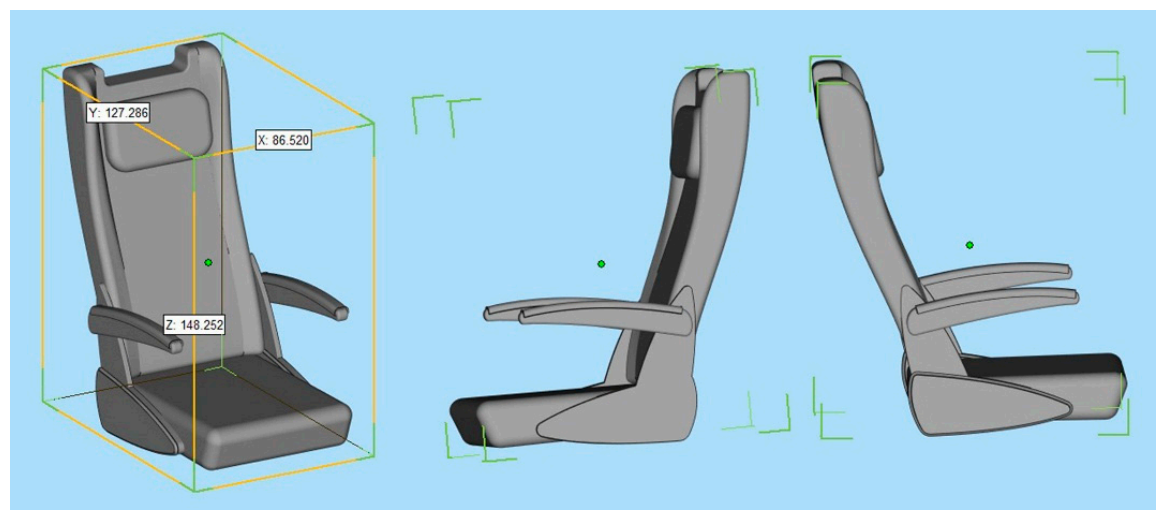

Figure 15. The 3-D printed drawing of the seat form sample 3. 


\section{Research Limitations and Discussions}

This paper takes the seat image design of high-speed train as the research case, and this method is also applicable to other industrial product design, interaction design, service design and user research fields. Based on the experimental framework of Kansei Engineering, the Kansei image perceived by users is transformed into product design elements, interaction design elements or service design elements, etc. Moreover, by establishing quantitative and qualitative decision models of product image design, the integrated decision system of product image design is obtained. The research system can evaluate the perceptual experience of users, make the designers work more efficiently and accurately, and promote and optimize the process of product design, interaction design, service design and user research by predicting the Kansei image of products.

The next work is to further explore the product image design integrated decision system based on Kansei Engineering, combined with product environment elements. The integrated decision system will be expanded and upgraded in the future. With the change of society and the passing of time, the perceptual cognition of users will change constantly. The evaluation database of experimental subjects and the Kansei image database of products in the integrated decision system of product image design need to be adjusted and updated timely to ensure that the integrated decision system of product image design can go with the tide of development and the user environment for any new product market.

In the intelligent application environment, more high and new technologies are developing rapidly. Therefore, the development trend of the product image design integrated decision system will be more intelligent, accurate, digital and systematic. Meanwhile, in the establishment process of experimental database, the design concept, 3-D model and rendering of product design will be further optimized and promoted [18]. Therefore, the intelligent research of product image design integrated decision system has broad prospects and far-reaching research significance.

\section{Conclusions}

In this paper, we have proposed a methodology of product image design integrated decision system based on Kansei Engineering theory. Firstly, Quantification Theory Type I is used to effectively identify the influential design elements that affect the product image, and the product image design qualitative decision model is constructed. Secondly, according to GRA-Fuzzy theory, the GRA-Fuzzy logic sub-models of influential design elements for the product image are constructed. Then, combining with the utility optimization model, the product image design quantitative decision model is built up. Finally, the product image design integrated decision system is constituted to analyze, decide and evaluate the product image design. The result shows that the system is effective in predicting the product images and optimizes the product image design process.

To illustrate the product image design integrated decision system, we have performed an experimental study on train seat design. The result demonstrates that the system has a better performance for improving the product image design. The designers can effectively plan the product design for the specific product image to meet the user perceptual expectation. Although the train seat design is used as a case in this paper, the methodology can be applied to other user-oriented product designs with multiple design elements. Along with the development of modern science and technology innovation and development, the product image design integrated decision system involved with multiple product design elements and product images can be further improved and developed in the future.

Author Contributions: Conceptualization, L.X. and X.Y.; methodology, L.X.; software, L.X.; validation, L.X. and Y.Z.; formal analysis, L.X.; investigation, Y.Z.; resources, X.Y.; writing-original draft preparation, L.X.; writing-review and editing, L.X., X.Y. and Y.Z.; visualization, L.X. and Y.Z.; supervision, X.Y.; project administration, X.Y.; funding acquisition, L.X. and X.Y. All authors have read and agreed to the published version of the manuscript.

Funding: This research was funded by the Major Program of Beijing Municipal Science and Technology Commission under grant number D16111000110000. 
Acknowledgments: We would like to thank the 60 subjects, 10 product designers, 10 design experts for their participation and assistance in the experimental study. We also appreciate the editor and anonymous reviewers for their valuable comments.

Conflicts of Interest: The authors declare no conflict of interest.

\section{References}

1. Sarah, L.; John, V. Industrial Design for Modern Life by Danielle Shapiro. Technol. Cult. 2018, 59, 490-492.

2. Zheng, P.; Yu, S.; Wang, Y.; Zhong, R.Y.; Xu, X. User-experience based product development for mass personalization: A case study. Procedia CIRP 2017, 63, 2-7. [CrossRef]

3. Li, Y.; Shieh, M.D.; Yang, C.C. A posterior preference articulation approach to Kansei engineering system for product form design. Res. Eng. Des. 2019, 30, 3-19. [CrossRef]

4. Wei, W.; Tian, Z.; Peng, C.; Liu, A.; Zhang, Z. Product family flexibility design method based on hybrid adaptive ant colony algorithm. Soft Comput. 2018, 23, 509-520. [CrossRef]

5. Lei, N.; Moon, S.K. A Decision Support System for market-driven product positioning and design. Decis. Support Syst. 2015, 69, 82-91. [CrossRef]

6. Yang, S.S.; Ong, S.K.; Nee, Y.C. A Decision Support Tool for Product Design for Remanufacturing. Procedia CIRP 2016, 40, 144-149. [CrossRef]

7. Yang, W.; Su, J.; Qiu, K.; Zhang, X.; Zhang, S. Research on Evaluation of Product Image Design Elements Based on Eye Movement Signal. In Proceedings of the International Conference on Human-Computer Interaction, Paphos, Cyprus, 2 September 2019; Springer: Cham, Switzerland.

8. Shieh, M.D.; Li, Y.; Yang, C.C. Comparison of multi-objective evolutionary algorithms in hybrid Kansei engineering system for product form design. Adv. Eng. Inform. 2018, 36, 31-42. [CrossRef]

9. Wu, Y.; Cheng, J. Continuous Fuzzy Kano Model and Fuzzy AHP Model for Aesthetic Product Design: Case Study of an Electric Scooter. Math. Probl. Eng. 2018, 2, 1-13. [CrossRef]

10. Florio, S.; Jones, N.K. Unrestricted Quantification and the Structure of Type Theory. Philos. Phenomenol. Res. 2019, 8, 1-21. [CrossRef]

11. Chang, H.-C.; Chen, H.-Y. Exploration of Action Figure Appeals Using Evaluation Grid Method and Quantification Theory Type I. Eurasia J. Math. Sci. Technol. Educ. 2016, 13, 1445-1459. [CrossRef]

12. Das, R.; Ball, A.K.; Roy, S.S. Optimization of E-jet Based Micro Manufacturing Process Using Grey Relation Analysis. Mater. Today Proc. 2018, 5, 200-206. [CrossRef]

13. Ivanov, O.A.; Ivanova, V.V.; Saltan, A.A. Likert-scale questionnaires as an educational tool in teaching discrete mathematics. Int. J. Math. Educ. 2018, 1, 1-9. [CrossRef]

14. Rikalovic, A.; Cosic, I.; Labati, R.D.; Piuri, V. Intelligent Decision Support System for Industrial Site Classification: A GIS-Based Hierarchical Neuro-Fuzzy Approach. IEEE Syst. J. 2018, 12, $2970-2981$. [CrossRef]

15. Duffuaa, S.O.; Gaaly, A.E. A multi-objective optimization model for process targeting with inspection errors using 100\% inspection. Int. J. Adv. Manuf. Technol. 2017, 88, 1-14. [CrossRef]

16. Lin, Y.C.; Wei, C.C. A hybrid consumer-oriented model for product affective design: An aspect of visual ergonomics. Hum. Factors Ergon. Manuf. Serv. Ind. 2017, 27, 17-29. [CrossRef]

17. Xue, L.; Yi, X.; Lin, Y.C.; Drukker, J.W. A method of the product form design and color design of train seats based on GRA-Fuzzy theory. J. Eng. Technol. 2018, 6, 517-536.

18. Zhang, D.; Li, Z.; Qin, S.; Han, S. Optimization of Vibration Characteristics of Fused Deposition Modeling Color 3D Printer Based on Modal and Power Spectrum Method. Appl. Sci. 2019, 9, 41-54. [CrossRef] 